\title{
A cluster-based feature selection method for image texture classification
}

\author{
Abbas F. H. Alharan ${ }^{1}$, Hayder K. Fatlawi², Nabeel Salih Ali ${ }^{3}$ \\ ${ }^{1}$ Computer Department, College of Education for Girls, University of Kufa, Iraq \\ ${ }^{2,3}$ Information Technology Research and Development Centre/University of Kufa, Iraq
}

\begin{tabular}{l} 
Article Info \\
\hline Article history: \\
Received Nov 22, 2018 \\
Revised Jan 21, 2019 \\
Accepted Feb 23, 2019 \\
\hline
\end{tabular}

Keywords:

Classification

Feature evaluation

Feature extraction

Feature selection

Image texture features.

K-means clustering

\begin{abstract}
Computer vision and pattern recognition applications have been counted serious research trends in engineering technology and scientific research content. These applications such as texture image analysis and its texture feature extraction. Several studies have been done to obtain accurate results in image feature extraction and classifications, but most of the extraction and classification studies have some shortcomings. Thus, it is substantial to amend the accuracy of the classification via minify the dimension of feature sets. In this paper, presents a cluster-based feature selection approach to adopt more discriminative subset texture features based on three different texture image datasets. Multi-step are conducted to implement the proposed approach. These steps involve texture feature extraction via Gray Level Cooccurrence Matrix (GLCM), Local Binary Pattern (LBP) and Gabor filter. The second step is feature selection by using K-means clustering algorithm based on five feature evaluation metrics which are infogain, Gain ratio, oneR, ReliefF, and symmetric. Finally, K-Nearest Neighbor (KNN), Naive Bayes (NB) and Support Vector Machine (SVM) classifiers are used to evaluate the proposed classification performance and accuracy. Research achieved better classification accuracy and performance using KNN and NB classifiers that were $99.9554 \%$ for Kelberg dataset and $99.0625 \%$ for SVM in Brodatz-1 and Brodatz-2 datasets consecutively. Conduct a comparison to other studies to give a unified view of the quality of the results and identify the future research directions.
\end{abstract}

Copyright $\odot 2019$ Institute of Advanced Engineering and Science. All rights reserved.

\section{Corresponding Author:}

Nabeel Salih Ali,

Information Technology Research and Development Centre/University of Kufa,

Kufa, P.O. Box (21), Najaf Governorate, Iraq.

Email: nabeel@uokufa.edu.iq

\section{INTRODUCTION}

In the digital era, Data mining concerned with the substantial problems that often comprise an enormous number of features. Since several features are frequent and irrelevant, not all of them are important, which may minimize the correctness of an algorithm such as classification algorithms. Attribute selection or Feature selection (FS) seeks to solve such a problem by choosing only a small subset of informative features from the original massive set of features [1]. In another meaning, FS aims to select a subset of $q$ related features from a set of $\mathrm{p}$ features, where $\mathrm{q}<\mathrm{p}$. Ignoring the redundant and irrelevant features can eliminate the dimensionality of data and improve the performance of numerous machine learning approaches [2]. Feature selection has been pointed to several functions such as 1) selecting a subset of informative features from the original set of features, 2) Obtaining new features from the unique features by transformation, which called as feature extraction [3]-[5]. In contrast with FS selection, the feature extraction relies on the ability of descriptor method to capture the optimal feature subset; the physical semantic of the original features may be missed [4], [6]. 
Also, FS is deemed one of the essential activities in diverse fields like computer vision, pattern recognition, machine learning, and classification issues. Concerning computer vision, most of its applications transact with visual features so-called textures [7]. Mainly, the texture is a distinct feature which is used to describe the inner intensity distribution of an image. Better classification accuracy can be obtained in numerous texture recognition works dues based on their systems combine different multi descriptors to extract the features [3], [8]. The current work concentrates on the texture features for the image. FS methods can be classified into three types, Filter-based, wrapper-based, and hybrid-based (filter and wrapper) [9]. The filter-based method is looking for a subset in the feature domain by specific search strategy and then evaluating such subset by an independent metric. While the wrapper-based method uses a specified learning algorithm to assess the feature subset. Finally, the hybrid method benefits from the merits of both filter and wrapper-based methods. In the current work, the wrapper- based method will be focused.

In this paper, presents an approach to select more discriminative subset texture features from three different texture image datasets. To implement the proposed method, three steps should be achieved. The first step aims to extract the texture features, which are the Gray Level Co-occurrence Matrix (GLCM), Local Binary Pattern (LBP) and Gabor feature from the dataset of texture images. Afterward, feature selection is fulfilled using K-means clustering algorithm after assessing the set of texture features by using five attribute evaluation metrics, which are infogain, Gain ratio, oneR, ReliefF and symmetric. Finally, the classification step is accomplished using three classifiers like K-Nearest Neighbor (KNN), Naive-Bayes (NB) and Support Vector Machine (SVM) to evaluate the effectiveness of the proposed approach. The remainder of the paper will be organized as follows. Reviews kinds of literature regarding the feature selection in Section 2. Section 3 describes all steps of the research methodology. The experiments are presented in Section 4. Finally, Section 5 provides the conclusions and future work.

\section{RELATED WORKS}

In this section, the literature of some learning algorithm-based feature selection methods is reviewed. Feature selection is an active process to get rid of the features that negatively influence the accuracy and speedup of learning [10]. Different prior studies have been suggested to develop the feature selection methods.

Zhang et al. [11] presented a glioma grading system that is relied on combining histogram of the texture features of multi-parametric MRI. (32) Existed statistical metrics are used to cover the histogram features. The texture features are extracted using radiomics tool. After oversampling all tumor features using SMOTE technique, a combination of (25) common classifiers and (8) feature selection methods are applied and assessed by LOOCV strategy. The proposed system achieved higher classification accuracy with SVM classifier [11]. Alongside, as stated in [12], a classification system for wood defect images is introduced by Tong et al. The system employed six different methods to extract the color and texture features of the images. The texture features are captured by GLCM, LBP, DWF, and Gabor while the color histogram and color coherence vector methods are adapted to extract the color features. A PSO selects a subset of the extracted features set, Tabu search and Ranker then classified by SVM, KNN, Random forest, and J48. The best classification result obtained by polynomial-SVM and GLCM features using the Ranker as a feature selection method [12]. Likewise, Siricharoen et al., [13] proposed a plant's disease detection system which is performed in a mobile-cloud environment. The system is based on many color and shape methods to describe images of leaf plant to discover the disease. The critical features are selected by SFS method after ranking them using three methods that are namely ACC, ReliefF, and mRMR. The experiment results proved that the combination of color and shape features listed by mRMR has the best classification performance and results respectively (using Linear SVM) [13]. In addition to, a study presented in [14] for multi-textural features classification; the texture features are basic statics (mean, standard deviation, range, and median), Tamura, GLCM, and LBP. PCA reduces the dimensionality of the features then passed to a classifier algorithm. Results achieved better classification accuracy using SVM classifier that was (94.14\%) and (96\%) for Bodatz and Outex datasets consecutively [14]. Furthermore, Pape and Kluhas in [15] are developed a system by depending on the geometric image features extracted by their IAP software for three datasets (A1, A2, and A3). The conducted system aims to predict the leaf count of Rosette plan images. The most regular features are gained by using ReliefF and Ranker method. The authors compared all regression approaches in Weka to get the superior classifier results, where the lowest error attained by IBk for A1, DecisionTable for A2 and SubSpaceRandom for A3 [15]. As well, the study [16] that is utilized the Gabor filter technique to express the statistical, topological and geometric features of the signature images in a signature verification system. The crucial features selection is performed using the rough set theory to reduce the signatures. The results had shown that the superior accuracy of various classifier methods is obtained when they applied among the minimum selected features [16]. Moreover, object-based pattern recognition system for an agricultural area 
in the mapping of UAV is presented by Ma et al. [17]. To express the map images, many spectral, shape, textural (by GLCM and GLDV) are extracted. The study objectives are to assess the influence of the existed feature selection methods of SVM and RF classifiers. (8) Feature selection methods are implemented in the presented work, filter-based (Gain ratio, ReliefF, CFS, Chi-square, and SVM-RFE), wrapper-based (RF and SVM), and RF as an embedded method. The overall results proved that SVM-RFE is the top for both classifiers, while the CFS is the evaluation method for feature subset [17]. Besides, a work that introduced by Obaidullah et al., [18] which is Handwritten Script Identification (HIS) system for document images. The features of the images are extracted by applying four transform-based techniques namely DCT, RT, FFT, and DT. The performance of the eight different feature selection methods (BFS, ES, GS, GSS, LFSS, PSOS, RS, and GAS) are compared utilizing five well-known classifiers (BayesNet, LIBLinear, LMT, MLP, and PART) for the Average Accuracy Rate (AAR) and Model Building Time (MBT) [18]. The best AAR was in the case of LMT + ES, while there was many cased for best MBT.

In this paper, conduct a cluster-based feature selection approach to adopt more discriminative subset texture features based on three different texture image datasets. Several steps are made to implement the producer approach like texture feature extraction, feature selection, and multi-classifier are used to evaluate the proposed classification performance and accuracy.

\section{METHODOLOGY OF THE PROPOSIING APPROACH}

To perform the proposed approach, numerous steps can be implemented which are: feature extraction, feature selection, classification, and the evaluation of the classification accuracy and performance. Figure 1 illustrates all the steps of the proposed method.

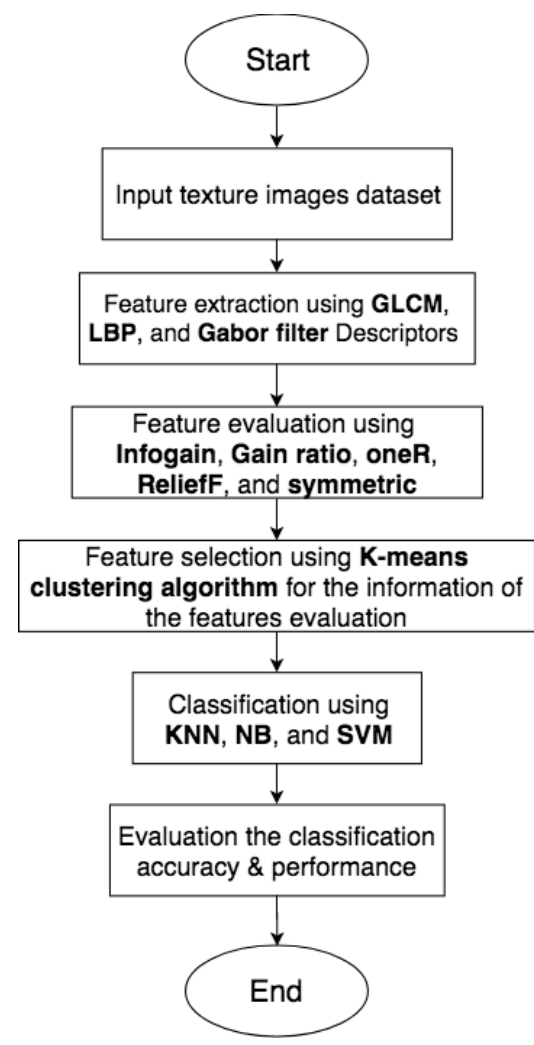

Figure 1. The methodology of the proposed approach

\subsection{Feature Extraction}

Diverse features are utilized to describe the characteristics of the image texture. These features are Gray-Level Co-occurrence Matrix (GLCM), Local Binary Pattern (LBP), and Gabor filter. The combination of the extracted features from these three methods will be considered as the original set of the features, which will be passed into the next step. The texture feature extraction methods are discussed as the following: 


\subsubsection{Gray-Level Co-occurrence Matrix (GLCM)}

It is a popular method for texture feature extraction. GLCM observes the relation between two adjacent pixels (second-order texture) of a grayscale image concerning the tone or grayscale intensity and the spatial distance (d) in certain direction $(\theta)$, where the first pixel (i) called the reference and the second (j) is the neighbor pixel [19]. The GLCM is in dimension $\mathrm{N}^{*} \mathrm{~N}, \mathrm{~N}$ is the number of gray levels in an image. Therefore, the GLCM would be Pd,(i,j). After creating the GLCM, many statistical features can be extracted by using various formulas as mentioned in [20]. In the current work, (13) features are extracted from GLCM based on in the following equations see Table 1 .

Table 1. The Equations of GLCM Features

\begin{tabular}{cccc}
\hline No. & \# of features & Feature name & Equation \\
\hline 1 & 1 feature & Contrast & $\sum_{i, j}|i-j|^{2} p(i, j)$ \\
2 & 1 feature & Correlation & $\sum_{i, j} \frac{\left(i-\mu_{i}\right)\left(i-\mu_{j}\right) p(i, j)}{\sigma_{i} \sigma_{j}}$ \\
4 & 1 feature & Energy & $\sum_{i, j}(p(i, j))^{2}$ \\
5 & 1 feature & Homogeneity & $\sum_{i, j} \frac{p(i, j)}{1+|i-j|}$ \\
6 & $\begin{array}{l}8 \text { features since the variance is calculated for the } \\
\text { columns of } 8 \text { gray levels GLCM }\end{array}$ & Entropy & $\sum_{i, j}^{\text {Variance }}$ \\
\hline
\end{tabular}

Where $(i, j)$ is a value of GLCM when $i$ and $j$ are references to rows and a column respectively. $\mu_{-} i$ and $\mu \mathrm{j}$ are the mean values according to $\mathrm{i}$ and $\mathrm{j}$ references respectively, respect to the 1 and 2 :

$$
\begin{aligned}
& \mu_{i}=\sum_{i, j} p(i, j) i \\
& \mu_{j}=\sum_{i, j} p(i, j) j \\
& \sigma_{i} \text { and } \sigma_{j} \text { are the standard deviation }(\sqrt{\text { Variance }}) \text { of values for } i \text { and } j \text { references respectively. }
\end{aligned}
$$

\subsubsection{Local Binary Pattern (LBP)}

LBP is a powerful method for getting the texture description of an image, introduced by Ojala et al. in [21]. The important property of LBP is no arbitrary regarding variation and rotation in the local gray-scale. Simply, the central pixel gc is subtracted from each gray value of the closed circular neighborhood ( $\mathrm{P}=0, \ldots, \mathrm{P}-$ 1) that are surround the gc. 3 is to calculate the LBP:

$$
\operatorname{LBP}(P, R)=\sum_{p=0}^{p-1} s\left(g_{p}-g_{c}\right) 2^{p}
$$

Where $\mathrm{P}$ represents the number neighborhood pixels, gp is the gray-level value of pixel $\mathrm{P}$ in the radius $\mathrm{R}, \mathrm{s}(\mathrm{x})$ $=1$ if $\mathrm{x}>=0$; means $\mathrm{gp}>=\mathrm{gc}$, and $\mathrm{s}(\mathrm{x})=0$ if $\mathrm{x}<0$; means $\mathrm{gp}<\mathrm{gc}$.

\subsubsection{Gabor Filter}

Gabor filter (GF) is extensively used to extract the texture features of an image by capturing the energy of whole an image [22]. GF can be defined as a combination of wavelets; each one captures the energy at a specific frequency (f) and orientation (k). The total number of $f$ and $k$ used in experiments equals six and four respectively. In each frequency, six features for each mean and standard deviation will produce, which are snipped into 8 features as in. So the total number of Gabor filter for each image (I) is 32 .

Suppose the set of the extracted features was I, so I(i,j) is the $j$-th features of the $i$-th instance, where $\mathrm{i}=1$ to $\mathrm{n}, \mathrm{j}=1$ to $\mathrm{m}, \mathrm{n}$ is the number of instances(images) in the dataset, $\mathrm{m}$ is the number of features for each instance. In other words, the rows in the set of features represent the instances, while the columns are considered as the features its self. 


\subsection{Features Selection}

The objective of this step is the dimensionality reduction of the extracted features before the classification process. Use of the feature selection methods contributes positively to the performance of the classifier. This step consists of two sub-steps, which are feature evaluation, and clustering.

\subsubsection{Feature Evaluation}

For better understanding about the quality of the selected features, five feature evaluation methods using Ranker-based search method which are applied on the features set (I), and all methods are performed using Weka (feature selection mode) [23]. Description of these feature evaluators is listed in Table 2. After performing the evaluators on set $\mathrm{I}$, the matrix of new information ( $\mathrm{R}$, which includes the data of evaluation) will be produced. The dimensionality of $\mathrm{R}$ is $\mathrm{r} \times \mathrm{s}$, where $\mathrm{r}$ is the number of features; $\mathrm{s}$ is the number of evaluator methods. The information of a new matrix $(\mathrm{R})$ has to be clustered by the k-mean algorithm (see next sub-step).

Table 2. Description of the Feature Evaluators

\begin{tabular}{|c|c|}
\hline Evaluator method & Description \& implementation \\
\hline Information Gain & $\begin{array}{l}\text { It measures the ration of the gained information to the essential information by taking into account the } \\
\text { size and number of branches to reduce the bias [24]. It is implemented by Weka } \\
\text { (weka.attributeSelection.InfoGainAttributeEval). }\end{array}$ \\
\hline Gain Ratio & $\begin{array}{l}\text { It is an improvement for the information gain metric. Gain ratio tries to solve the problem of bias in } \\
\text { the information gain measure over the feature selection process in the case of large values[25]. It is } \\
\text { implemented byWeka (weka.attributeSelection.GainRatioAttributeEval). }\end{array}$ \\
\hline OneR & $\begin{array}{l}\text { Evaluates the quality of features utilizing the OneR classifier, Implemented by Weka } \\
\text { (weka.attributeSelection.OneRAttributeEval). }\end{array}$ \\
\hline ReliefF & $\begin{array}{l}\text { Evaluates the quality of all features by repeatedly training the randomly sampled data of instance with } \\
\text { considering the feature value and the class of this value [26]. It is implemented by Weka } \\
\text { (weka.attributeSelection.ReliefFAttributeEval). }\end{array}$ \\
\hline $\begin{array}{l}\text { Symmetrical } \\
\text { Uncertainty }\end{array}$ & $\begin{array}{l}\text { Evaluates the quality of a feature by observing the symmetrical uncertainty measurement about the } \\
\text { class. It is implemented by Weka (weka.attributeSelection.SymmetricalUncerAttributeEval). }\end{array}$ \\
\hline
\end{tabular}

\subsubsection{Clustering by K-means algorithm}

$\mathrm{K}$-means is one of the popular algorithms that used for data clustering and analysis; its standard idea was proposed by Stuart Lloyd firstly in 1957. This algorithm clusters the data object into a predetermined number of clusters $(\mathrm{k})$, each object belongs to a particular cluster from calculating the sum of the square distance between each point and the centroid of that cluster. In the current work, the role of K-means clustering algorithm is to separate the data of $R$ into two clusters $(K=2)$. One of them involves more related features, while the other cluster includes the noisy features.

\subsection{Classification Algorithms}

In this study, three common different classifiers are carried out, namely Support Vector Machine (SVM), Naive Bayes (NB) and K-Nearest Neighbor (KNN). They are purposely chosen to ensure the proposed method does not bias for a specific classifier. Besides, several works in different texture-based image systems that proved the effectiveness of these classifiers as in [12], [14], [15], [27]-[29]. The implantation and more information about the aforementioned classification algorithms are detailed in [23], [30].

\section{RESULTS AND DISCUSSION}

\subsection{Dataset Preparation}

The proposed approach has experimented with three datasets of texture images, all the images in the datasets are grayscale images and without rotation. The first dataset is Kelberg [31], which includes (28) texture classes and each class consists of $(160)$ images with size $(576 \times 576)$ pixels for each one. While the second and third datasets are formed from Brodatz dataset [28]. The original of the datasets consist of (112) images, and each one is $(640 \times 640)$ pixels as a class. (20) Classes are randomly selected and resampled once into (100) non-overlapping sub-images with size $(64 \times 64)$ pixels (Brodats-1), and once again into $(64)$ nonoverlapping sub-images with size $(80 \times 80)$ pixels $($ Brodatz-2). The detailed information of the three datasets is summarized in Table 3. 
Table 3. The Datasets Information

\begin{tabular}{cccc}
\hline Dataset & Classes & Instances & Dimensions \\
\hline Kelberg & 28 & 4880 & $576 \times 576$ \\
Brodatz-1 & 20 & 2000 & $64 \times 64$ \\
Brodatz-2 & 20 & 1280 & $80 \times 80$ \\
\hline
\end{tabular}

\subsection{Feature Selection Method}

Since five evaluation metrics are applied to each dataset features, new data matrix consist of $\mathrm{n}$ instances $(n=$ dataset features) and $r$ features ( $r=$ evaluation metrics) is transformed. K-means clustering algorithm implementation of the data of feature evaluation creates two clusters, and one holds the goodness features and the irrelevant features in the other cluster. The results of the K-means clustering of the three datasets are shown in the Tables 4-6 and Figures 2-4. From Tables 4-6, notice that the features of Kylberg, Brodatz-1, and Brodatz-2 datasets have been reduced from (301) to (43), (81), and (77) features respectively. Furthermore, cluster 0 is the best cluster in all datasets clustering results.

Table 4. Final Cluster Centroids (Kelberg Dataset)

\begin{tabular}{cccc}
\hline feature & $\begin{array}{c}\text { Full data } \\
(301)\end{array}$ & $\begin{array}{c}\text { Cluster 0 } \\
(43)\end{array}$ & $\begin{array}{c}\text { Cluster 1 } \\
(258)\end{array}$ \\
\hline Infogain & 0.3333 & 0.6682 & 0.2775 \\
Gain ratio & 0.3177 & 0.6733 & 0.2584 \\
oneR & 0.2601 & 0.5594 & 0.2102 \\
ReliefF & 0.3334 & 0.497 & 0.3061 \\
symmetric & 0.2735 & 0.4229 & 0.2486 \\
\hline
\end{tabular}

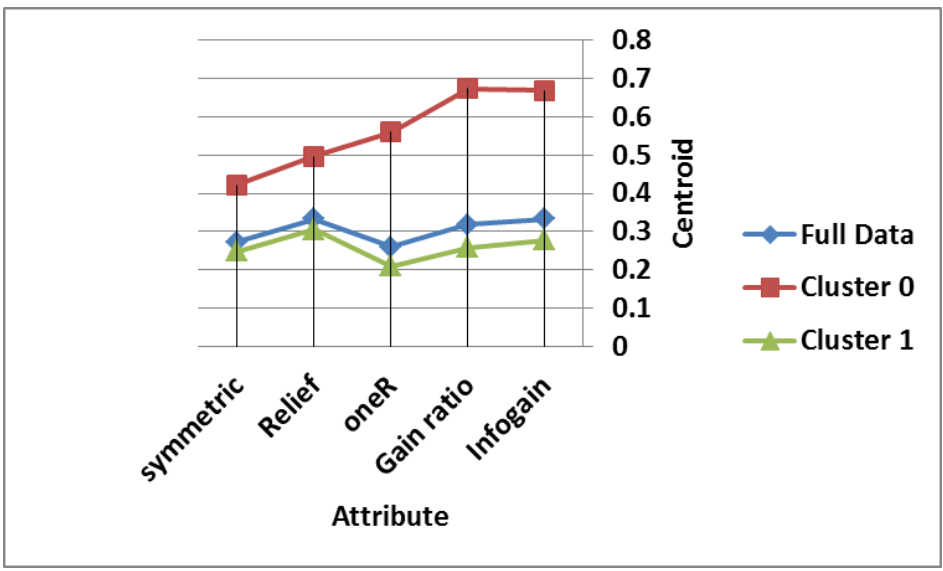

Figure 2. The result of clustering for the Kylberg dataset

Table 5. Final Cluster Centroids (Brodatz-1 Dataset)

\begin{tabular}{cccc}
\hline feature & $\begin{array}{c}\text { Full data } \\
(301)\end{array}$ & $\begin{array}{c}\text { Cluster 0 } \\
(81)\end{array}$ & $\begin{array}{c}\text { Cluster 1 } \\
(220)\end{array}$ \\
\hline Infogain & 0.3691 & 0.6642 & 0.2605 \\
Gain ratio & 0.2737 & 0.6169 & 0.1473 \\
oneR & 0.2926 & 0.5867 & 0.1843 \\
ReliefF & 0.2492 & 0.483 & 0.1631 \\
symmetric & 0.1743 & 0.3368 & 0.1144 \\
\hline
\end{tabular}

Table 6. Final Cluster Centroids (Brodatz-2 Dataset)

\begin{tabular}{cccc}
\hline feature & $\begin{array}{c}\text { Full data } \\
(301)\end{array}$ & $\begin{array}{c}\text { Cluster 0 } \\
(77)\end{array}$ & $\begin{array}{c}\text { Cluster 1 } \\
(224)\end{array}$ \\
\hline Infogain & 0.3808 & 0.6554 & 0.2864 \\
Gain ratio & 0.3091 & 0.661 & 0.1882 \\
oneR & 0.313 & 0.6214 & 0.207 \\
ReliefF & 0.2889 & 0.3279 & 0.2755 \\
symmetric & 0.2125 & 0.2172 & 0.2109 \\
\hline
\end{tabular}




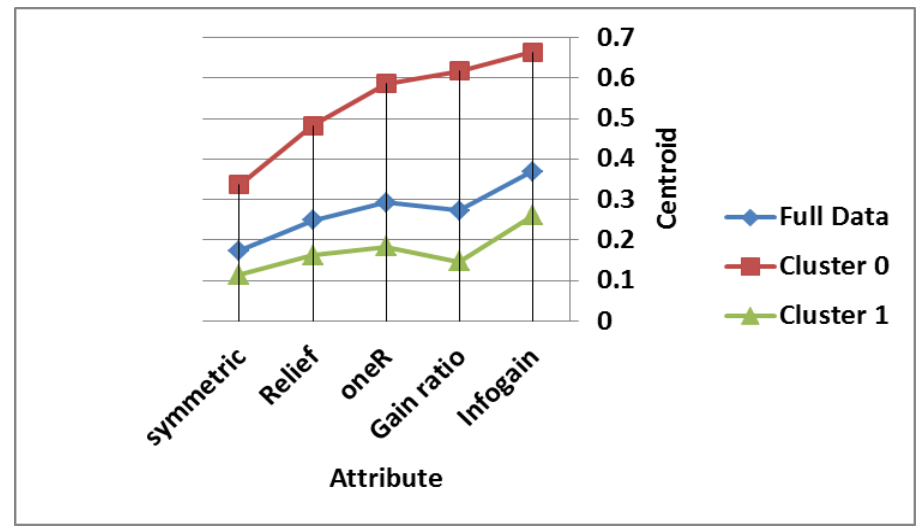

Figure 3. The result of clustering for the Brodatz-1 dataset

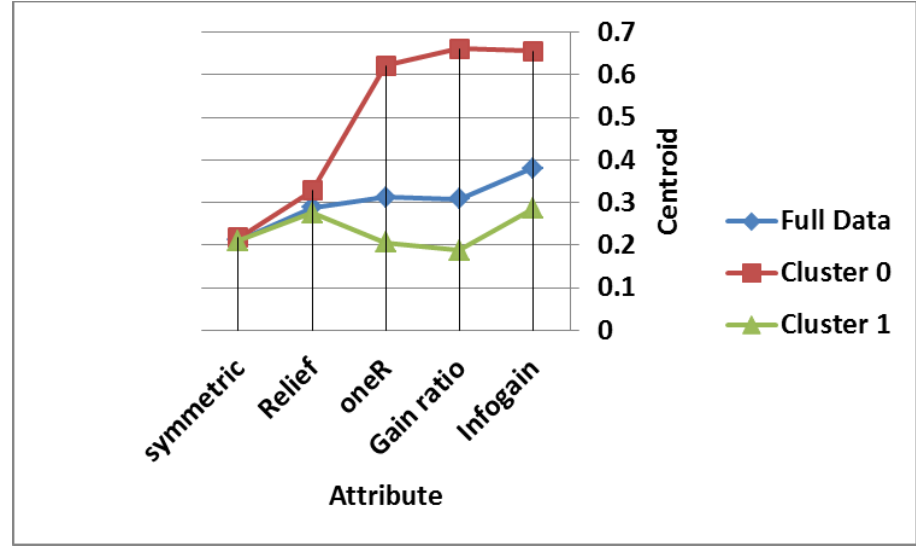

Figure 4. The result of clustering for the Brodatz-2 dataset

\subsection{Classification Accuracy}

Feature selection or attribute selection is an essential factor to locate the performance of the classifier. Wherefore, it has been an active research topic in data mining and machine learning [32]. To demonstrate the effectiveness of the proposed feature selection, three classifier methods (KNN, NB, and SVM) are applied to the mentioned datasets before and after the feature selection.

Table 7. Classification Performance of the Three Datasets (Kelberg, Brodatz-1, And Brodatz-2) Using KNN,

\begin{tabular}{lllllll}
\multicolumn{7}{c}{ NB and SVM Classifiers } \\
\hline \multicolumn{1}{c}{ dataset } & \multicolumn{2}{c}{ Kylberg } & \multicolumn{2}{c}{ Brodatz-1 } & \multicolumn{2}{c}{ Brodatz-2 } \\
\hline Classifier & $\begin{array}{l}\text { Before } \\
\text { selection }\end{array}$ & After selection & $\begin{array}{l}\text { Before } \\
\text { selection }\end{array}$ & After selection & Before selection & After selection \\
KNN & $99.5536 \%$ & $99.9554 \%$ & $95.5 \%$ & $98.8 \%$ & $97.1875 \%$ & $\mathbf{9 8 . 9 8 4 4} \%$ \\
NB & $97.0313 \%$ & $99.9554 \%$ & $98 \%$ & $97.35 \%$ & $98.3594 \%$ & $98.125 \%$ \\
SVM & $99.9554 \%$ & $99.3973 \%$ & $98.5 \%$ & $99 \%$ & $98.5938 \%$ & $\mathbf{9 9 . 0 6 2 5 \%}$ \\
\hline
\end{tabular}

Table 7 shown the performance that achieved by KNN and NB classifiers among the selected features is the best for Kylberg dataset. For Brodatz-1 and Brodatz-2 datasets, the best performance obtained by SVM classifier. Better performance of the classification on the selected features means that those features have positive impacts. The correctly classified instances of the complete features datasets are slightly higher than of the selected features datasets, as in SVM (99.9554) on Kylberg, NB (98\%) on Brodatz-1 and NB (98.3594\%) on Brodatz-2. Figures 5-7 show the results of the classifiers for the three datasets respectively using NN, NB, and SVM for each of complete and selected features. 


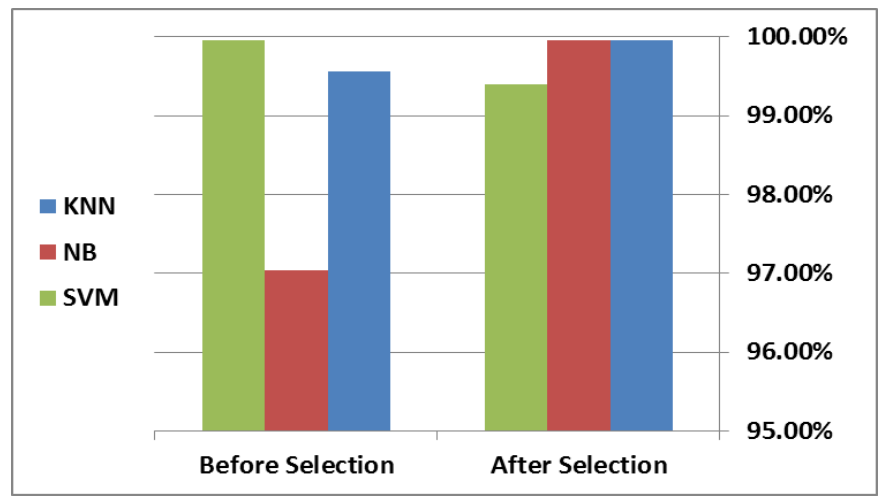

Figure 5. The result of classification methods (KNN, NB, and SVM) for the Kylberg dataset (before and after feature selection)

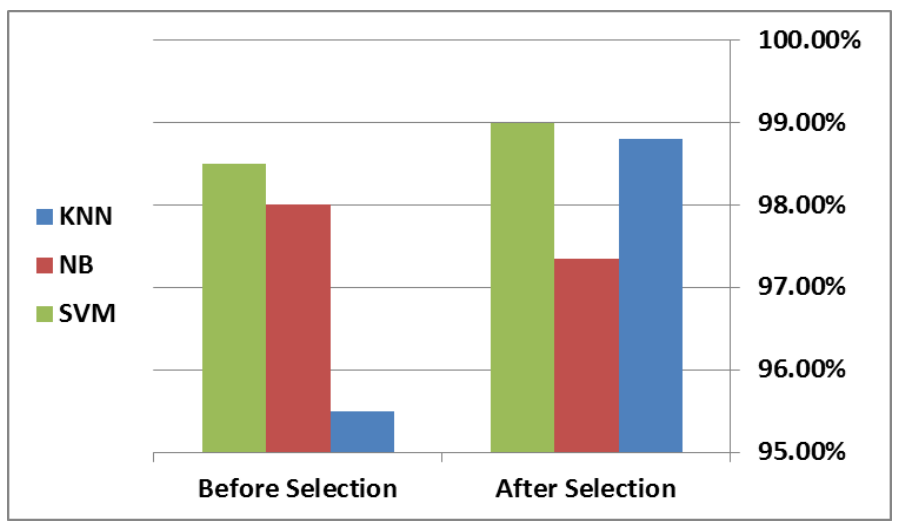

Figure 6. The result of classification methods (KNN, NB, and SVM) for the Brodatz-1 dataset (before and after feature selection)

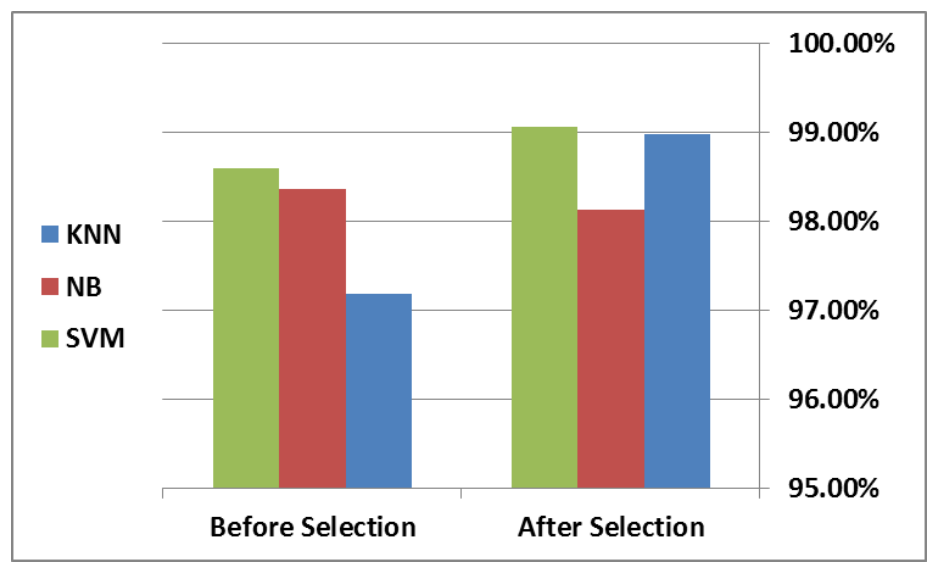

Figure 7. The result of classification methods (KNN, NB, and SVM) for the Brodatz-2 dataset (before and after feature selection)

The comparison between the proposed method and other former works regarding the classification performance is shown in Tables 8 and 9 for Kylberg and Brodatz datasets respectively. 
Table 8. The Comparison Between the Proposed Method and Other Existed Work Concerning the Classification Performance (Kylberg Dataset)

\begin{tabular}{lcc}
\hline Research paper & Classifier & Performance (\%) \\
\hline Kaya et al. (2015)[27] & KNN & 99.64 \\
Zheng et al. (2016)[33] & VisualNet & 97.8 \\
Al-Sahaf et al. (2017)[28] & KNN & 91.1 \\
Calzada-Ledesma et al. (2018) [29] & Linear SVM & 99.22 \\
Proposed method & KNN & $\mathbf{9 9 . 9 5 5 4}$ \\
Proposed method & NB & $\mathbf{9 9 . 9 5 5 4}$ \\
Proposed method & SVM & 99.3973 \\
\hline
\end{tabular}

Table 9. The Comparison Between the Proposed Method and Other Existed Work Regarding the Classification Performance (Brodatz Dataset)

\begin{tabular}{lcc}
\hline Research paper & Classifier & Performance (\%) \\
\hline Al-Sahaf et al. (2017)[28] & KNN & 90.9 \\
Kaya et al. (2015)[27] & KNN, BN & 98.51 \\
Al-Sahaf et al. (2017)[34] & KNN & 93 \\
Yang et al. (2017) [14] & SVM & 94.14 \\
Proposed method & KNN & 98.9844 \\
Proposed method & NB & 98.125 \\
Proposed method & SVM & $\mathbf{9 9 . 0 6 2 5}$ \\
\hline
\end{tabular}

For the Kylberg dataset, the results proved that the proposed approach has better classification performance with (99.9554\%) for each of KNN and NB. Besides, the results of the Brodatz dataset revealed that the proposed method outperforms the other existing methods with a performance $(99.0625 \%)$ using SVM classifier.

\section{CONCLUSIONS AND FUTURE RESEARCH DIRECTIONS}

In this study, three texture image datasets are used for classification purpose. Three texture feature sets among them are extracted from original images; they are GLCM, LBP, Gabor filter Statistics respectively. We used a K-Means clustering algorithm for feature selection by using five attributes evaluation metrics. These metrics are infogain, Gain ratio, oneR, ReliefF and symmetric respectively. Three classifiers such as SVM, NB, and KNN are used to classify the aforementioned three feature sets extracted from Kelberg, Brodatz-1, and Brodatz-2 datasets respectively. And the results accuracy was achieved high ratios like $99.9554 \%$ by KNN and NB, and $99 \%$ by SVM among the Kelberg dataset is better than the methods that are produced in [24], [31], [25] and [26] for both KNN and NB classifiers (see Table 8). And the result of the Brodatz-1 and Brodatz-2 databases is better than previous studies that are [25], [24] and [32] by using SVM classifier with accuracy ration $99.0625 \%$ given by the proposed approach (see Table 9 ). In the future, we intend to implement the proposed method with other datasets in different fields as well will focus on the additional classifiers to be used to improve accuracy and performance as well.

\section{REFERENCES}

[1] B. Xue, et al., "A survey on evolutionary computation approaches to feature selection," IEEE Transactions on Evolutionary Computation, vol. 20, pp. 606-626, 2016.

[2] I. Guyon and A. Elisseeff, "An introduction to variable and feature selection," Journal of machine learning research, vol. 3, pp. 1157-1182, 2003.

[3] A. Jain and D. Zongker, "Feature selection: Evaluation, application, and small sample performance," IEEE Transactions on pattern analysis and machine intelligence, vol. 19, pp. 153-158, 1997.

[4] J. Tang, et al., "Feature selection for classification: A review," Data classification: Algorithms and applications, p. 37, 2014.

[5] Anita Mohanty, Sudipta Mahapatra, Urmila Bhanja. (2019). Traffic congestion detection in a city using clustering techniques in VANETs. Indonesian Journal of Electrical Engineering and Computer Science, 13(2),pp. 884-891.

[6] Md. Zakir Hossain, Md. Nasim Akhtar, R.B. Ahmad, Mostafijur Rahman. (2019). A dynamic K-means clustering for data mining. Indonesian Journal of Electrical Engineering and Computer Science, 13(2), pp. 521-526.

[7] Wan Aida Nadia Wan Abdullah, Naimah Yaakob, R. Badlishah Ahmad, Mohamed Elshaikh Elobaid, , Siti Asilah Yah. (2019). Impact of clustering in AODV routing protocol for wireless body area network in remote health monitoring system. Indonesian Journal of Electrical Engineering and Computer Science, 13(2), pp. 689-695.

[8] Fatlawi, H.K., Alharan, A.F.H., Ali, N.S. (2018). An Efficient Hybrid Model for Reliable Classification of High Dimensional Data Using K-Means Clustering And Bagging Ensemble Classifier. Journal of Theoretical and Applied Information Technology, 96(24), pp. 8379- 8398. 
[9] H. Liu and L. Yu, "Toward integrating feature selection algorithms for classification and clustering," IEEE Transactions on knowledge and data engineering, vol. 17, pp. 491-502, 2005.

[10] C.-H. Chen, "Feature selection for clustering using instance-based learning by exploring the nearest and farthest neighbors," Information Sciences, vol. 318, pp. 14-27, 2015.

[11] X. Zhang, et al., "Optimizing a machine learning based glioma grading system using multi-parametric MRI histogram and texture features," Oncotarget, vol. 8, p. 47816, 2017.

[12] H. Tong, et al., "Evaluation of feature extraction and selection techniques for the classification of wood defect images," J. Eng. Appl. Sci, vol. 12, pp. 602-608, 2017.

[13] P. Siricharoen, et al., "Texture and shape attribute selection for plant disease monitoring in a mobile cloud-based environment," in Image Processing (ICIP), 2016 IEEE International Conference on, 2016, pp. 489-493.

[14] T. Yang, et al., "Combining multiple feature for texture image classification," in Image and Signal Processing, BioMedical Engineering and Informatics (CISP-BMEI), 2017 10th International Congress on, 2017, pp. 1-5.

[15] J.-M. Pape and C. Klukas, "Utilizing machine learning approaches to improve the prediction of leaf counts and individual leaf segmentation of rosette plant images," Proceedings of the Computer Vision Problems in Plant Phenotyping (CVPPP), pp. 1-12, 2015.

[16] S. Das and A. Roy, "Signature verification using rough set theory based feature selection," in Computational Intelligence in Data Mining-Volume 2, ed: Springer, 2016, pp. 153-161.

[17] L. Ma, et al., "Evaluation of feature selection methods for object-based land cover mapping of unmanned aerial vehicle imagery using random forest and support vector machine classifiers," ISPRS International Journal of GeoInformation, vol. 6, p. 51, 2017.

[18] S. M. Obaidullah, et al., "Handwritten Indic Script Identification from Document Images-A Statistical Comparison of Different Attribute Selection Techniques in Multi-classifier Environment," in Proceedings of the Second International Conference on Computer and Communication Technologies, 2016, pp. 491-500.

[19] M. Benčo and R. Hudec, "Novel method for color textures features extraction based on GLCM," Radioengineering, vol. 16, p. 65, 2007.

[20] R. M. Haralick, "Statistical and structural approaches to texture," Proceedings of the IEEE, vol. 67, pp. 786-804, 1979.

[21] T. Ojala, et al., "Multiresolution gray-scale and rotation invariant texture classification with local binary patterns," IEEE Transactions on pattern analysis and machine intelligence, vol. 24, pp. 971-987, 2002.

[22] J. G. Daugman, "Complete discrete 2-D Gabor transforms by neural networks for image analysis and compression," IEEE Transactions on acoustics, speech, and signal processing, vol. 36, pp. 1169-1179, 1988.

[23] M. Hall, et al., "The WEKA data mining software: an update," ACM SIGKDD explorations newsletter, vol. 11, pp. 10-18, 2009.

[24] J. R. Quinlan, "Induction of decision trees," Machine learning, vol. 1, pp. 81-106, 1986.

[25] J. Han, et al., Data mining: concepts and techniques: Elsevier, 2011.

[26] M. Robnik-Šikonja and I. Kononenko, "Theoretical and empirical analysis of ReliefF and RReliefF," Machine learning, vol. 53, pp. 23-69, 2003.

[27] Y. Kaya, et al., "Two novel local binary pattern descriptors for texture analysis," Applied Soft Computing, vol. 34, pp. 728-735, 2015.

[28] H. Al-Sahaf, et al., "Automatically evolving rotation-invariant texture image descriptors by genetic programming," IEEE Transactions on Evolutionary Computation, vol. 21, pp. 83-101, 2017.

[29] V. Calzada-Ledesma, et al., "Evolutionary Design of Problem-Adapted Image Descriptors for Texture Classification," IEEE Access, vol. 6, pp. 40450-40462, 2018.

[30] I. H. Witten, et al., Data Mining: Practical machine learning tools and techniques: Morgan Kaufmann, 2016.

[31] G. Kylberg, "The Kylberg Texture Dataset v. 1.0, Centre for Image Analysis, Swedish University of Agricultural Sciences and Uppsala University, External report (Blue series) No. 35," ed, 2011.

[32] M. Tan, et al., "Learning sparse svm for feature selection on very high dimensional datasets," in Proceedings of the 27th international conference on machine learning (ICML-10), 2010, pp. 1047-1054.

[33] H. Zheng, et al., "Deep learning for surface material classification using haptic and visual information," IEEE Transactions on Multimedia, vol. 18, pp. 2407-2416, 2016.

[34] H. Al-Sahaf, et al., "A Multitree Genetic Programming Representation for Automatically Evolving Texture Image Descriptors," in Asia-Pacific Conference on Simulated Evolution and Learning, 2017, pp. 499-511. 